\title{
Strategien in der Behandlung von angeborenen Handfehlbildungen
}

\author{
Strategies in Treatment of Congenital Hand Malformation
}

\author{
Autor \\ W. Hülsemann \\ Institut \\ Handchirurgie, Katholisches Kinderkrankenhaus Wilhelmstift, Hamburg
}

\section{Schlüsselwörter \\ - angeborene Handfehl- bildung \\ - Behandlungsstrategie \\ - Zehentransplantation}

Key words

- congenital anomalies of the hand

- treatment strategy

- toe-to-hand transfer

eingereicht $\quad 30.8 .2015$

akzeptiert 7.1.2016

Bibliografie

DOI http://dx.doi.org/

10.1055/s-0042-100457

Handchir Mikrochir Plast Chir 2016; 48: 2-9

(c) Georg Thieme Verlag KG

Stuttgart · New York

ISSN 0722-1819

Korrespondenzadresse Dr. Wiebke Hülsemann

Handchirurgie

Katholisches Kinderkranken-

haus Wilhelmstift

Liliencronstraße 130

22149 Hamburg

w.huelsemann@kkh-

wilhelmstift.de

\section{Zusammenfassung \\ $\nabla$}

Das Ziel der Behandlung von Fehlbildungen ist eine Verbesserung der Funktion, um den Betroffenen weitestgehend ein eigenständiges Leben ohne fremde Hilfe zu ermöglichen. Da zunächst die Eltern über die Behandlung ihrer Kinder entscheiden, müssen sie vom Behandlungskonzept überzeugt sein. Bei Fehlbildungen muss auch bei gleichem Krankheitsbild mit unterschiedlichen pathologische Veränderungen gerechnet werden. Das erfordert unter Umständen intraoperativ ein Abändern des präoperativ geplanten Vorgehens. Aufgrund möglicher Befundveränderungen im weiteren Verlauf ist das Begleiten der Betroffenen bis zum Abschluss des Wachstums erforderlich. Im Artikel werden die Denkweise und das Entwickeln eines Therapiekonzeptes bei Handfehlbildungen beschrieben.

Fehlbildungen sind relativ selten. Sie treten bei 1-2\% der Neugeborenen auf. Bei jedem zehnten Kind mit einer Fehlbildung sind die oberen Extremitäten betroffen. Das entspricht einer Inzidenz von Arm- und Handfehlbildungen von 1:1000 Lebendgeborenen [1].

Im ersten Kontakt mit betroffenen Familien gilt es bei der Erstuntersuchung nicht nur die Erkrankung einzuordnen, sondern auch auf Fragen und Ängste der Eltern einzugehen. Die Eltern sind angespannt. Sie fragen nach Ursachen der Erkrankung und insbesondere die Mütter wollen wissen, ob sie Anteil an der Entstehung der Fehlbildungen haben. Sie fühlen sich schuldig und machen sich Sorgen um die Zukunft ihrer Kinder: ob sie im Leben zurechtkommen, ob sie in der Schule gehänselt werden und ob sie trotz der Fehlbildung glücklich werden können. Darauf gilt es, im ersten Gespräch einzugehen. Auch, um die Eltern in die Lage zu versetzen, den medizinischen Ausführungen folgen zu können.

\section{Abstract \\ $\nabla$}

The aim of the treatment of congenital hand anomalies is to achieve a functional improvement in order to enable patients to lead an independent life. As parents have to decide for their children early in life, they have to be convinced of the treatment proposal. One and the same disease may present with various individual pathologies, which may require intraoperative changes of the previously planned procedure. Because of possible changes during growth, doctors have to keep in touch with the patients up to the end of adolescence. This article presents our algorithm and surgical techniques for dealing with children with hand malformations.

\section{Ursachen}

$\nabla$

Ursachen sind meistens Veränderungen im Genmaterial (Mutationen), die spontan auftreten oder vererbt sein können. Selten werden Fehlbildungen von Teratogenen verursacht. Eine genetische Ursache liegt nahe, wenn mehrere Extremitäten, zusätzlich andere Organsysteme betroffen sind und die Familienanamnese positiv ist.

Das bekannteste Beispiel einer medikamenteninduzierten Fehlbildung ist das Schlafmittel Thalidomid (Contergan ${ }^{\circledR}$ ), das Ende der 1950er Jahre die Anzahl fehlgebildeter Babys in Mitteleuropa um über das 10 -fache ansteigen ließ. Es verursacht eine Reduktionsfehlbildung der oberen Extremitäten bei Einnahme zwischen 41. und 44. Tag post menstruationem und der unteren Extremitäten bei Einnahme zwischen dem 44. und 48. Tag [2]. Heute sehen wir in der Handchirurgie medikamenteninduzierte Anomalien, wenn überhaupt, am häufigsten nach Einnahme des Antiepi- 
leptikums Valproinsäure, das zu einer radialen Reduktionsfehlbildung führen kann.

Die Kinder selber leiden zunächst nicht an ihrer Andersartigkeit. Im Gegensatz zu posttraumatischen Folgeschäden kennen sie es nicht anders, hadern zunächst nicht mit ihrem Zustand und kommen im Alltag erstaunlich gut zurecht. Das kann sich im Laufe der Sozialisation ändern. Schwedische Kollegen haben das Selbstwertgefühl und die Zufriedenheit von Jugendlichen mit geringer, kaum sichtbarer Fehlbildung und von Jugendlichen mit schweren, deutlich sichtbaren Fehlbildungen im Vergleich zu normalen Jugendlichen untersucht. Es wurden keine signifikanten Unterschiede zwischen diesen 3 Gruppen gefunden. Die Jungen mit leichter Fehlbildung zeigten ein etwas schlechteres Selbstwertgefühl als die übrigen. Die Jugendlichen mit schwerer Fehlbildung schnitten im Intelligenztest und in den schulischen Leistungen besser ab als die übrigen [3].

\section{Erstvorstellung \\ $\nabla$}

\section{Symptom oder Syndrom?}

Um die Frage nach einer möglichen Vererbbarkeit beantworten zu können, schauen wir bei der Erstuntersuchung, ob ein Symptom oder Syndrom vorliegt. Ein Symptom ist ein Krankheitszeichen. Ein Syndrom ein Symptomenkomplex, also eine Gruppe von Zeichen, die für ein Krankheitsbild charakteristisch sind. Z.B. finden sich beim Apert-Syndrom, der Akrozephalosyndaktylie, typische Kopf-und Gesichtsveränderungen sowie Handund Fußfehlbildungen. Die Syndaktylien sind dabei ein Symptom des Apert-Syndroms. Durch die gleichen Gesichtsveränderungen ähneln sich diese Kinder, unabhängig davon, ob sie aus einer europäischen oder afrikanischen Familie stammen. Diese Ähnlichkeit bei unterschiedlichen Herkunftsfamilien und Ethnien nennen wir eine syndromale Ähnlichkeit.

\section{Klinische Untersuchung}

Um die Defizite bei der körperlichen Untersuchung von Säuglingen im ersten Lebensjahr abschätzen zu können, untersuchen wir die passive Beweglichkeit der Gelenke und schätzen die aktive Beweglichkeit ab. Ein exaktes Messen der aktiven Beweglichkeit ist bei Kleinkindern mangels Kooperation nicht möglich. Hilfreich bei der Untersuchung ist das Beachten von Furchen. Fehlen die Furchen über Gelenken, wurden die Kinder ohne aktive Beweglichkeit in diesen Gelenken geboren, und es wird sich auch keine aktive Beweglichkeit entwickeln ( $\bullet$ Abb. 1).

\section{Röntgenaufnahmen}

sind wichtig, um bestimmen zu können, welche Knochen angelegt bzw. hypoplastisch sind oder fehlen. Allerdings sind die Knochenenden in den ersten Lebensjahren noch knorpelig und daher nicht sichtbar. Die Gelenke entziehen sich in diesem Alter der radiologischen Beurteilung. Bei Fehlbildungen ist die Skelettentwicklung der betroffenen Extremität zusätzlich verzögert. Daher sind Röntgenbilder kurz nach der Geburt wenig aussagekräftig und im Alter von einem Jahr wesentlich besser beurteilbar. Bei Fehlbildungen können Fingerstrahlen fehlen, miteinander verschmolzen sein und Daumen wie Finger aufgebaut sein. Bei der Analyse von Röntgenbildern ist es ratsam, zuerst nach normal entwickelten Anteilen zu schauen, die eindeutig zugeordnet werden können. In der Regel kann der Radius gut von der Ulna unterschieden werden. So ist die Bestimmung der radialen und der ulnaren Seite möglich. Auf der radialen Seite erwartet

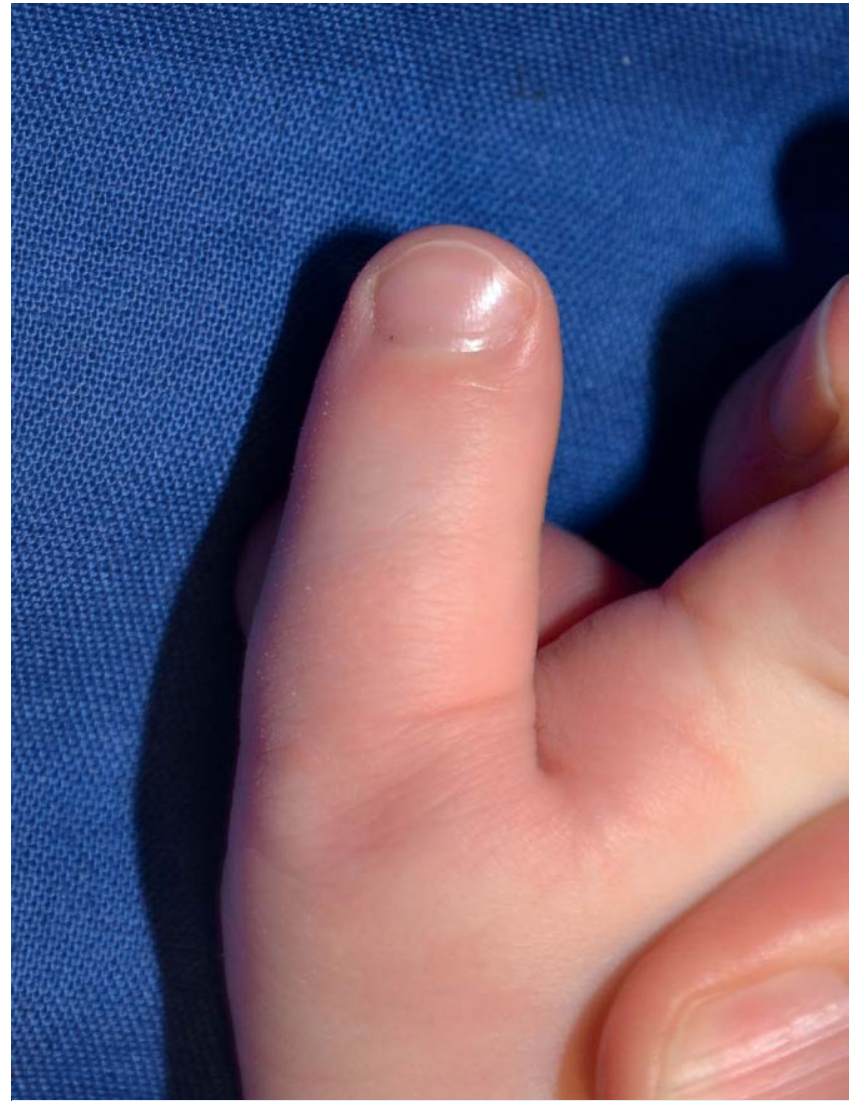

Abb. 1 Die fehlenden Furchen streckseitig über dem Daumenendgelenk eines 1-jährigen Jungen weisen auf eine angeborene Bewegungseinschränkung hin.

man den Daumenstrahl. Er unterscheidet sich von den Fingern durch seine 2-gliedrigkeit und dadurch, dass die Wachstumsfuge des ersten Mittelhandknochens proximal liegt. Der erste sichtbare Handwurzelknochen ist das Os capitatum. Es kalzifiziert am schnellsten und wird daher am frühesten sichtbar. In seiner Verlängerung liegt der Mittelfingerstrahl ( $\bullet$ Abb. 2). Neben der Röntgendiagnostik spielt heute auch die Ultraschalluntersuchung sowie in speziellen Fällen die Multi-Slice-Computertomografie-Angiografie zunehmend eine Rolle in der Diagnostik angeborener Handfehlbildungen.

\section{Therapieplan}

\section{$\nabla$}

\section{Ziel der Therapie}

Anhand der klinischen Untersuchung und der Analyse des Röntgenbildes wird der Therapieplan erstellt. Ziel jeder Therapie ist, eine Verbesserung der Handfunktion zu erzielen, das bilaterale Greifvermögen zu verbessern, um den Kindern die Bewältigung ihres Alltags zu erleichtern.

Selten können in der Fehlbildungschirurgie normale Hände geschaffen werden. Das ist nur bei Syndaktylien, beim Pollex rigidus und weitgehend beim einfachen Doppeldaumen möglich. Selten können wir das Aussehen maßgeblich verbessern. Das ist in seltenen Fällen wie bei der ulnaren Spalthand möglich. Durch Spaltverschluss nach Verlängerung des 5. Mittelhandknochens kann eine wesentlich normalere Handform und eine funktionell günstige breitere Mittelhand geschaffen werden ( $\bullet$ Abb. 3). In der Regel ist jedoch eine Normalisierung von Funk- 

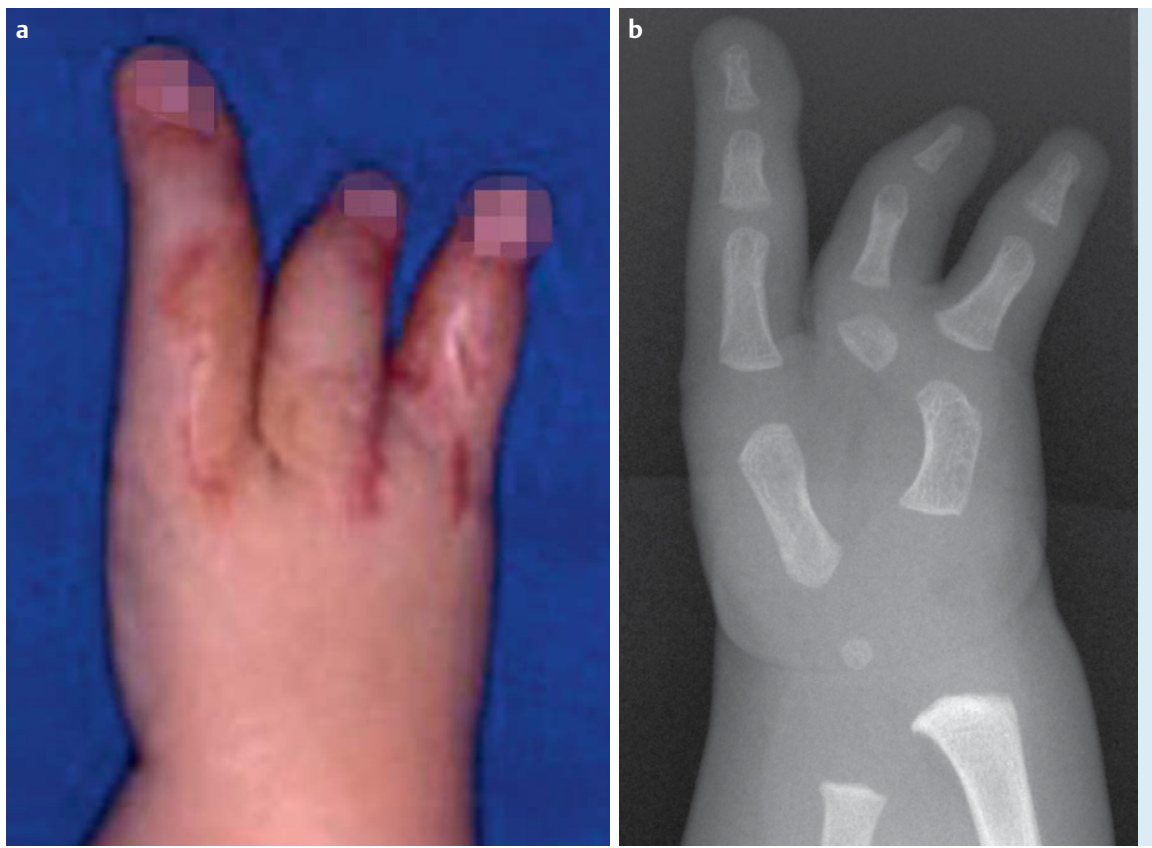

Abb. 2 Oligosyndaktylie nach auswärtiger Syndaktylietrennung. a Klinisch scheint eine 3-fingerhand vorzuliegen. b Die Röntgenaufnahme zeigt eine 2-fingerhand mit einem Doppeldaumen und einem Mittelfingerstrahl. Der Radius mit seiner breiteren Metaphyse liegt rechts. Auf dieser Seite besitzt der Mittelhandknochen eine proximale Wachstumsfuge. Es handelt sich also um den ersten Mittelhandknochen. Er setzt sich in einem Doppeldaumen fort. In Verlängerung des Os capitatum findet sich der Mittelfingerstrahl.
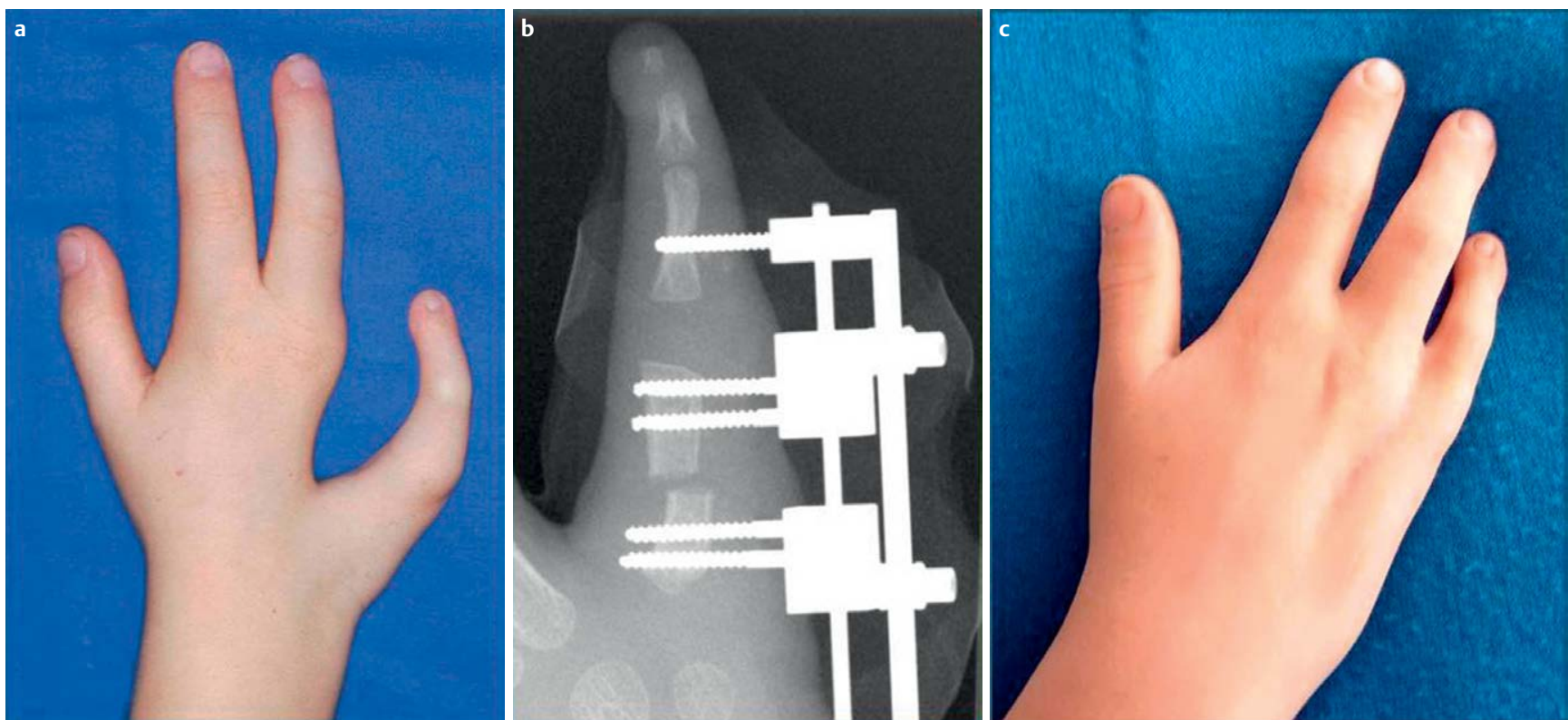

Abb. 3 Ulnare Spalthand: Die Handform wurde durch Verlängerung des ulnaren Fingerstrahls durch Kallusdistraktion mit anschließendem Spaltverschluss deutlich verbessert. a Situation präoperativ; b Kallusdistraktion; c Z. n. anschließendem Spaltverschluss.

tion und Aussehen nicht möglich, weil zu viele Anteile nicht angelegt sind. Ziel ist dann eine Verbesserung der Funktion mit möglichst gutem Aussehen zu erzielen. Das gelingt bei einer hochgradigen Daumenhypo- oder -aplasie durch eine Pollizisation des Zeigefingers gut. Der neue Daumen kann gut eingesetzt werden und die Hand sieht wesentlich normaler aus ( $\bullet$ Abb. 4). Im Vergleich zu einer normalen Hand fehlt nur die Sattelgelenksbeweglichkeit und die Kraft im Schlüsselgriff ist reduziert.

Je schwerer die Fehlbildung, umso aufwändiger ist in der Regel die Therapie. In manchen Fällen ist keine chirurgische Therapie sinnvoll. Ein Beispiel hierfür sind die verschiedenen Formen der Symbrachydaktylie.

Bei der monodaktylen Form ist ein Daumen vorhanden, die übrigen Finger fehlen. Zum Aufbau einer funktionierenden 2-fingerhand ist der Aufbau eines ulnaren Fingerstrahls durch eine freie Zehentransplantation ideal. Bei der peromelen Form mit Adaktylie und vorhandenem Karpus können 2 Fingerstrahlen durch eine 2-fache freie Zehentransplantation aufgebaut und so eine sensible Hilfshand zum bimanuellen Arbeiten geschaffen werden. Die Greiffunktion der betroffenen Hand ist aber deutlich schlechter, da die Finger durch die schlecht entwickelten extrinsischen Beuger und Strecker wenig beweglich und schwach sind. Beim völligen Fehlen der Hand kann chirurgisch keine Greiffunktion aufgebaut werden. Hier sind Hilfsmittel wie ein nach Maß gefertigter Lenkeraufsatz für das Fahrrad und Funktionsprothesen indiziert.

Bei Jugendlichen wird das Aussehen wichtiger. Manche fragen nach ästhetisch ansprechenderen Lösungen, die ihre veränderte Hand verstecken. Das ist mit Schmuckprothesen aus Silikon möglich. Sie sehen sehr echt aus, nehmen allerdings dem Stumpf 

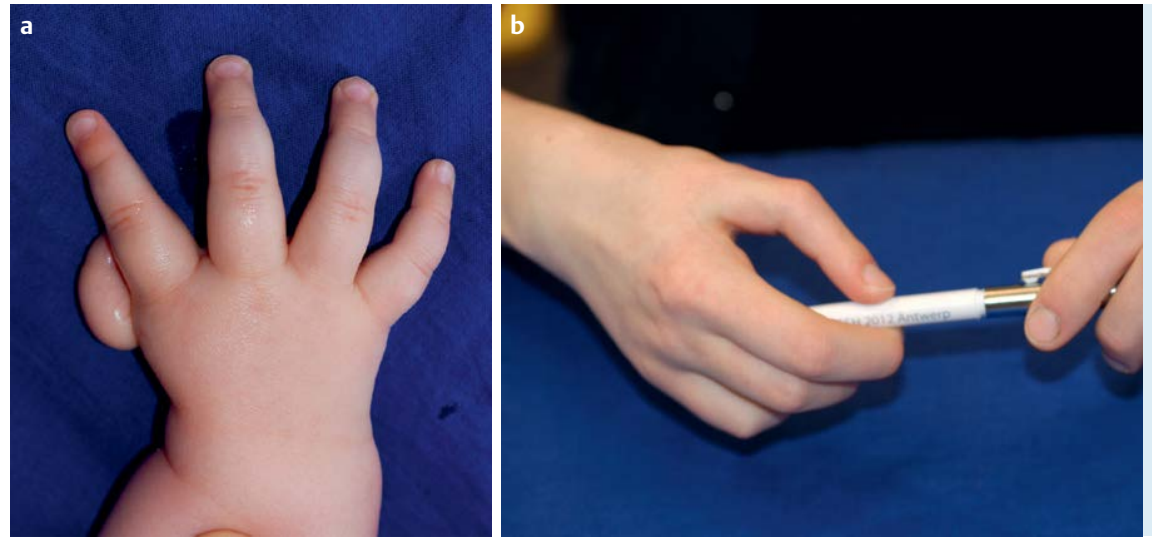

Abb. 4 Behandlung einer beidseitigen hochgradigen Daumenhypoplasie. a Rechte Hand präoperativ mit flottierendem Daumen. $\mathbf{b}$ Durch eine Pollizisation des Zeigefingers konnte eine gute Funktion und ein normaleres Aussehen erzielt werden.
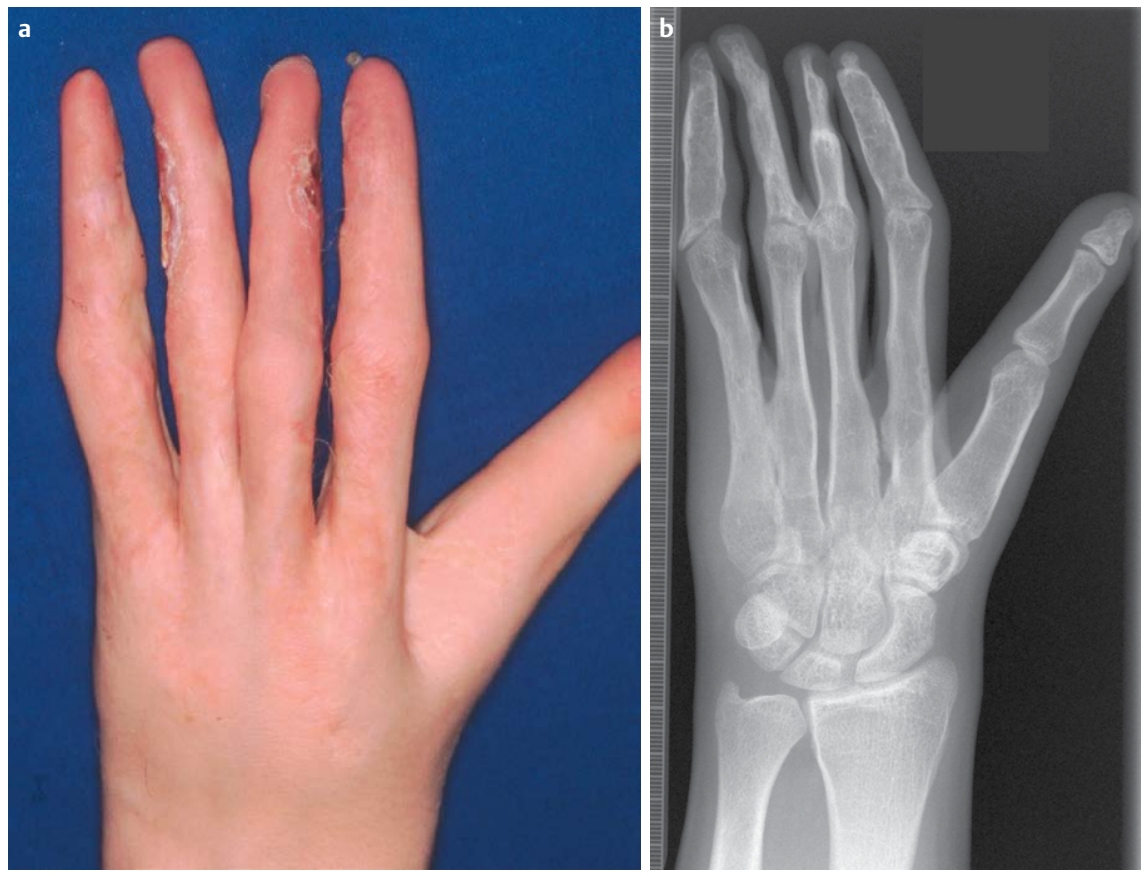

Abb. 5 Rechenförmige funktionslose Hand nach vielfacher Distraktion verkürzter Finger: a klinischer, $\mathbf{b}$ radiologischer Befund.

sein gutes Gefühl. So wird z. B. eine Tür von einem Schmuckprothesenträger nicht mehr mit dem Stumpf, sondern im Ersatz mit dem Ellenbogen geöffnet. Die gegebene Funktion wird durch die Schmuckprothese verschlechtert.

Skurrile Formen kann die kritiklose Fingerverlängerung bei der Symbrachydaktylie annehmen. @ Abb. 5 zeigt hierfür ein Beispiel. Durch extreme und wiederholte Fingerdistraktionen wurde zwar die Größe einer normalen Hand erreicht. Die Hand wurde jedoch zu einem funktionslosen unbeweglichen Rechen - ein ästhetisches und funktionelles Desaster mit zusätzlicher komplizierender Osteitis und Sequesterbildung des Ringfingers (๑ Abb. 5).

\section{Zeitlicher Ablauf}

Die erste operative Therapie ist in der 2. Hälfte des ersten Lebensjahres bzw. im zweiten Lebensjahr zu empfehlen. Es ist erstrebenswert, die Behandlung vor der Einschulung abzuschließen, damit die Kinder die Schule mit bestmöglicher Handfunktion beginnen können und um Fehlzeiten in der Schule zu vermeiden.

Sehr selten erzwingen akute Durchblutungsstörungen bei der Geburt eine notfallmäßige Operation. Beispiele für solche Situationen sind eine akute Abschnürung eines Fingers beim Schnürringsyndrom und das Kompartmentsyndrom des Neugebore- nen. Beim Schnürringsyndrom müssen die abschnürenden Fasern sofort entfernt werden. Beim Neugeborenenkompartmentsyndrom muss eine Kompartmentspaltung am Geburtstag erfolgen, um Schäden zu minimieren.

Die geplante erste Operation erfolgt zwischen 6 Monaten und 2 Jahren. Eine frühe Korrektur mit 6 Monaten ist bei Syndaktylien von Daumen und Zeigefinger ratsam, um den Säuglingen ein frühes Benutzen des Daumens zu ermöglichen, was ihre psychomotorische Entwicklung fördert. Bei Syndaktylien unterschiedlich langer Finger besteht die Gefahr sekundärer Gelenkkontrakturen, so am Ringfingermittelgelenk bei Syndaktylie zwischen Ring- und Kleinfinger. Um dies zu vermeiden, ist es ratsam, diese Finger nicht später als mit einem Jahr zu trennen.

Um die Zahl der Eingriffe und Narkosen zu reduzieren, sollen wenn möglich - 2 Extremitäten simultan durch 2 Teams operiert werden. Eine mehrschrittige operative Therapie ist indiziert, wenn eine Beeinträchtigung der Durchblutung durch eine zu ausgedehnte Operation zu befürchten ist. Bei einer Syndaktylie mehrerer benachbarter Finger ist eine 6-9 monatige Pause zwischen den Trennungen benachbarter Finger ratsam, weil dadurch das Risiko von Durchblutungs- und Wundheilungsstörungen verringert wird. Mit dem weiteren Wachstum können Stellungs- und Funktionsveränderungen eintreten. Diese Entwicklungen gilt es im Rahmen regelmäßiger 2- bis 4-jähriger 

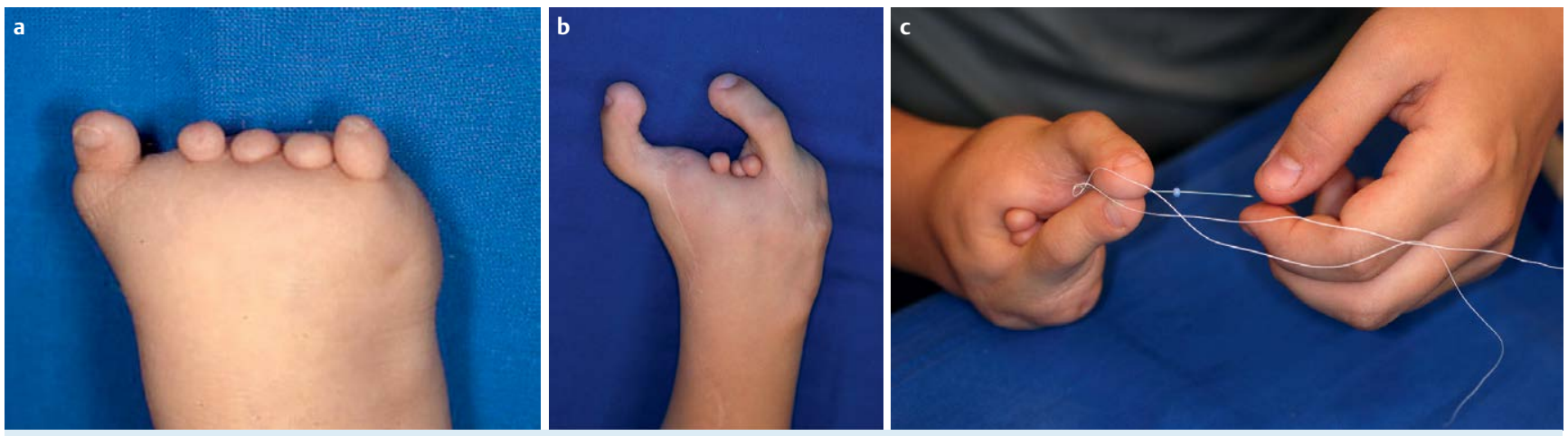

Abb. 6 a Peromele Symbrachydaktylie mit Adaktylie und den typischen Fingerrudimenten; b Aufbau einer 2-fingerhand durch 2-fache freie Zehentransplantation. Die mittleren Fingerrudimente wurden später entfernt; c Durch eine 2-fache freie Zehentransplantation wurde ein beidhändiges Greifen möglich.

Kontrollen zu überprüfen, um sie rechtzeitig zu erkennen und den optimalen Zeitpunkt für eine Therapie festlegen zu können.

\section{Hypo- und Aplasien}

In der Fehlbildungschirurgie sind wir oft mit der Situation konfrontiert, dass Anteile der Hand nicht angelegt oder für den Gebrauch zu kurz sind. Wir haben 3 Techniken, um Neues zu schaffen:

- die freie Zehentransplantation zum Fingeraufbau

- die Kallusdistraktion zur Knochenverlängerung

- und die quere Weichteildistraktion zur Weichteilvermehrung.

\section{Zehentransplantation}

Mindestvoraussetzung zum Greifen sind 2 Strahlen, die gegeneinander bewegt werden können. Eine klassische Indikation für eine Zehentransplantation besteht bei der monodaktylen Form der Symbrachydaktylie. Es ist ein Daumen, aber es sind keine weiteren Finger angelegt. Durch eine freie Zehentransplantation kann in einem operativen Schritt bereits im Vorschulalter ein stabiler Finger aufgebaut werden, der eine gute Länge und gute Sensibilität besitzt und mitwächst. Das Aussehen der Hand kann hierdurch nicht normalisiert werden. Die Form ist weiterhin stark verändert, die Funktion aber erheblich verbessert. Erstmalig wird ein beidhändiges Spielen und Arbeiten möglich, wobei die fehlgebildete Hand als sensible Hilfshand dient. Die Kinder können beidhändig mit Messer und Gabel essen, benötigen keine Hilfe beim Fleischschneiden, haben einen sensiblen Pinzettengriff mit einem 2-punkteunterscheidungsvermögen von 5 bis $6 \mathrm{~mm}$, eine weite Spanne von 8 bis $10 \mathrm{~cm}$, was das Greifen größerer Gegenstände ermöglicht. Sie können Flaschen gut öffnen und Schnürsenkel binden. Die Kraft im Spitzgriff beträgt im Durchschnitt 1-1,5 kg. Bei der peromelen Form kann nur durch einen 2-fachen Fingeraufbau ein bimanuelles Greifen aufgebaut werden $(\bullet$ Abb. 6a-c).

Hebedefekte an den Füßen sind nicht zu befürchten. Die Füße sind nach Entnahme des 2. Zehenstrahls schlank und gut geformt ( $\bullet$ Abb. 7). Sie sind voll belastbar und schmerzen auch bei sportlichen Aktivitäten nicht. Die Betroffenen können normales Schuhwerk tragen.

In Deutschland waren es Buck-Gramcko [4] und sein Team, die 1983 erstmalig erfolgreich bei dieser Fehlbildung eine mikrochirurgische Zehentransplantation durchführten. Die Zehentransplantation ist bei Fehlbildung technisch schwieriger durchzuführen als bei einem traumatischen Fingerverlust, weil die

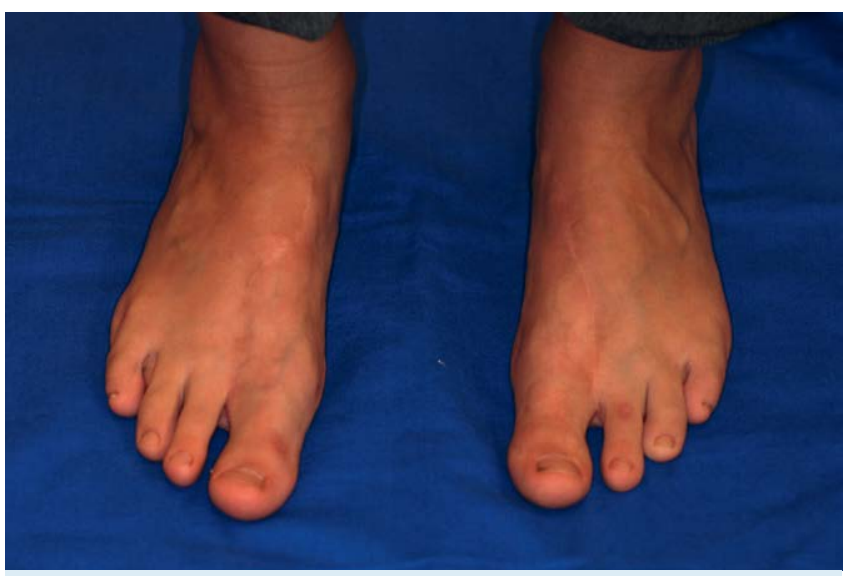

Abb. 7 Hebedefekt nach beidseitiger Entnahme der zweiten Zehen. Die Füße sind schlank und auch beim Sport voll belastbar.

Handstrukturen wesentlich kleiner sind und Anomalien besonders der Beuger und Nerven vorliegen.

Eine mögliche Option ist die Zehentransplantation auch bei der leichteren Form der Symbrachydaktylie mit unvollständig ausgebildetem Mittel- und Ringfinger (Symbrachydaktylie vom zentralen Defekttyp $\diamond$ Abb. 8). Dritter und vierter Finger wurden durch 2-fache Zehentransplantation auf die Grundglieder aufgebaut und die Hand so auch ästhetisch vervollständigt. Durch Stärkung des Grobgriffes können die Kinder kraftvoller zupacken und große Gegenstände, wie eine volle Flasche, wesentlich besser halten ( $\boldsymbol{O} \mathbf{A b b}$. 8b, $\mathbf{c})$

\section{Distraktion}

Mit der Distraktion von Knochen und Weichteilen haben wir eine zweite gut funktionierende Technik, um zu kurze Finger und Unterarme zu verlängern.

Die Weichteildistraktion ist eine sehr hilfreiche Technik in der Behandlung der radialen Klumphand. Sie erleichtert die Handgelenksaufrichtung.

Bei sehr engen komplexen Syndaktylien, die durch konventionelle Techniken nicht zu trennen sind, ist die quere Weichteildistraktion äußerst hilfreich. Klassische Indikation sind die verbliebenen Syndaktylien der Finger III und IV nach Korrektur von Löffelhänden beim Apert-Syndrom ( $\bullet$ Abb. 9a). Es verbleibt wenig eigene Haut, die Endglieder sind schmal und knöchern eng fusioniert. Durch eine quere Distraktion vor der eigentlichen Syndaktylietrennung können wesentlich breitere Endglie- 

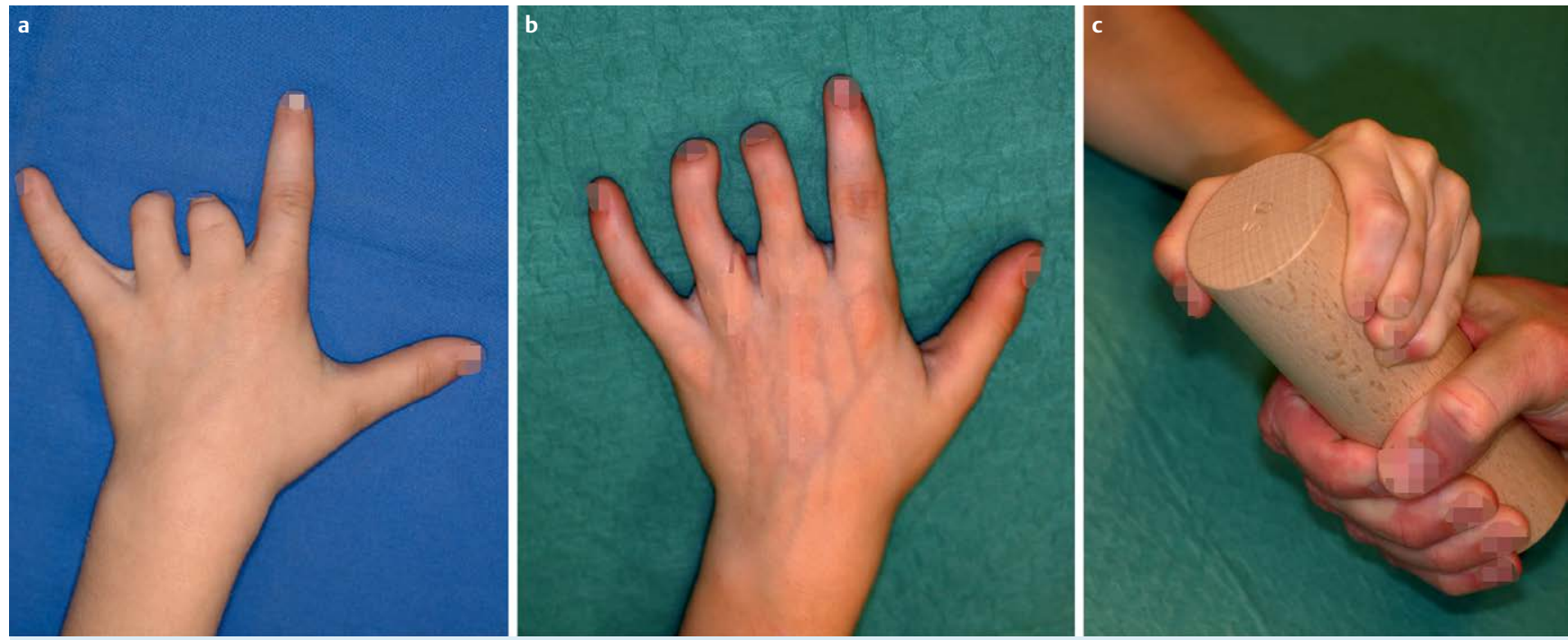

Abb. 8 Symbrachydaktylie vom zentralen Defekttyp: a präoperativ; b Vervollständigung der Hand durch Zehentransplantation auf die Grundglieder von Mittel- und Ringfinger; $\mathbf{c}$ die Kraft im Grobgriff ist deutlich verbessert: ein Holzklotz kann mit viel Kraft festgehalten werden.
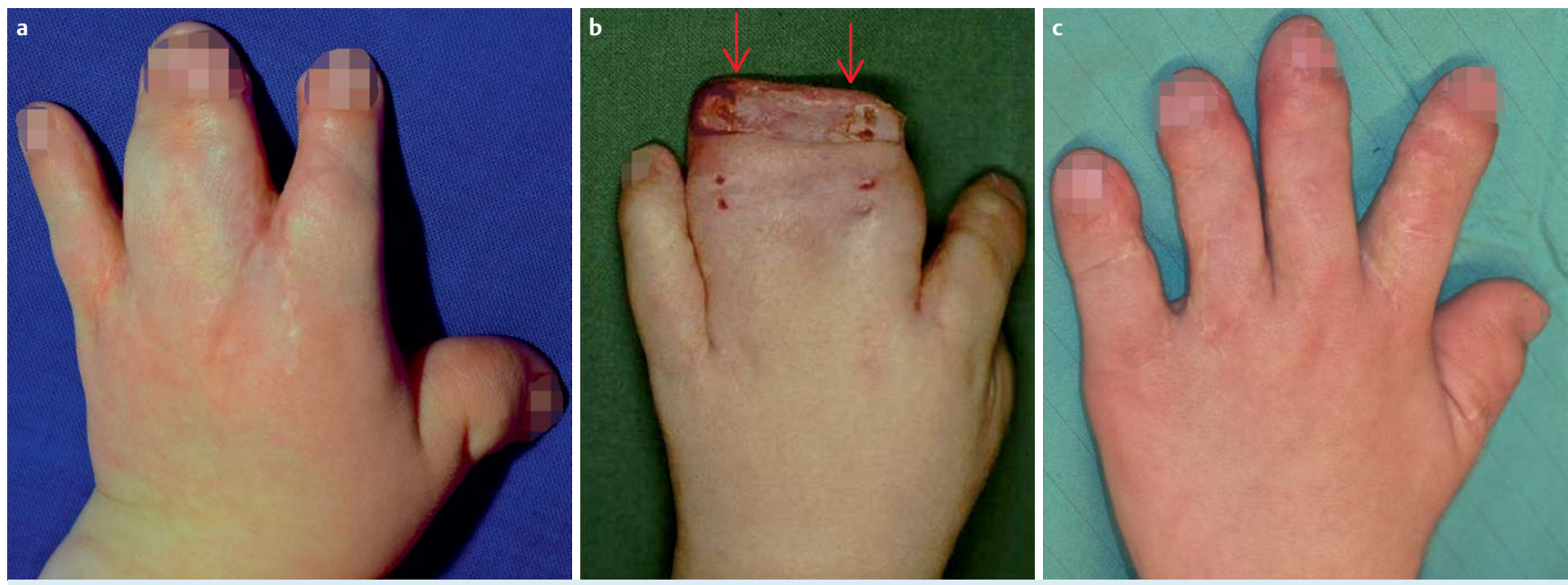

Abb. 9 Apert-Syndrom: Durch die quere Weichteildistraktion der Finger III und IV können die schmalen, eng zusammen liegenden Endglieder zu breiten gut geformten Endgliedern mit Nagelwällen und damit besseren Nagelanlagen geformt werden: a präoperativ; b nach Distraktion bei Abbau des Fixateurs. Die Pfeile markieren den Gewebszugewinn; c 3 Jahre postoperativ.

der mit breiteren Nagelanlagen von besserer Form und breitere Fingerbeeren erzielt werden $(\bullet \mathbf{A b b}$. 9b, c). Es werden weniger Hauttransplantate gebraucht. Ein erfreulicher Nebeneffekt ist eine wesentlich bessere Durchblutung, die auf eine verbesserte Angiogenese zurückzuführen ist. Sie erlaubt sogar ein komplikationsloses Trennen auch bei bilateral nicht angelegten palmaren Fingerarterien.

\section{Einfluss des Wachstums}

Bei verschiedenen Erkrankungen finden sich Deltaphalangen. Deltaphalangen sind Knochen mit atypischer bogenförmiger Wachstumsfuge. Die betroffenen Glieder sind trapezförmig, verkürzt und verbreitert. Deltaphalangen verursachen eine Seitdeviation der betroffenen Strahlen. Die Unterbrechung der klammerartigen Fuge oder ihre Resektion führt zu einer Aufrichtung und in manchen Fällen zu einem beeindruckenden Wachstumsschub. Diese Wachstumsmodulation hat sich besonders bei der
Klinodaktylie des Kleinfingers bewährt. Bei Unterbrechung der Deltaphalanx im Kleinkindalter kommt es bei Klinodaktylie in den folgenden Jahren zu einer spontanen Aufrichtung des Mittelgliedes. Ihre Unterbrechung im Kleinkindalter führt in den folgenden 5 Jahren zu einer spontane Aufrichtung. Eine aufwändige Korrektur in Form einer Korrekturosteotomie wird dadurch in vielen Fällen überflüssig.

Auch wenn wir die Veränderungen mancher Kinder nicht sicher einem Krankheitsbild zuordnen können, erlaubt das folgende Vorgehen das Erstellen eines Behandlungsplans: Wir analysieren zunächst die strukturellen Veränderungen und die fehlende Funktion. Dann überlegen wir, mit welchen der uns zur Verfügung stehenden Techniken wir die Funktion der Hände verbessern können. Am Beispiel eines 2-jährigen Mädchens soll dies erklärt werden ( $\bullet$ Abb. 10a,b): Welche Veränderungen liegen vor? Es sind radial zu viele Finger ausgebildet. Ulnar sind 2 Fin- 

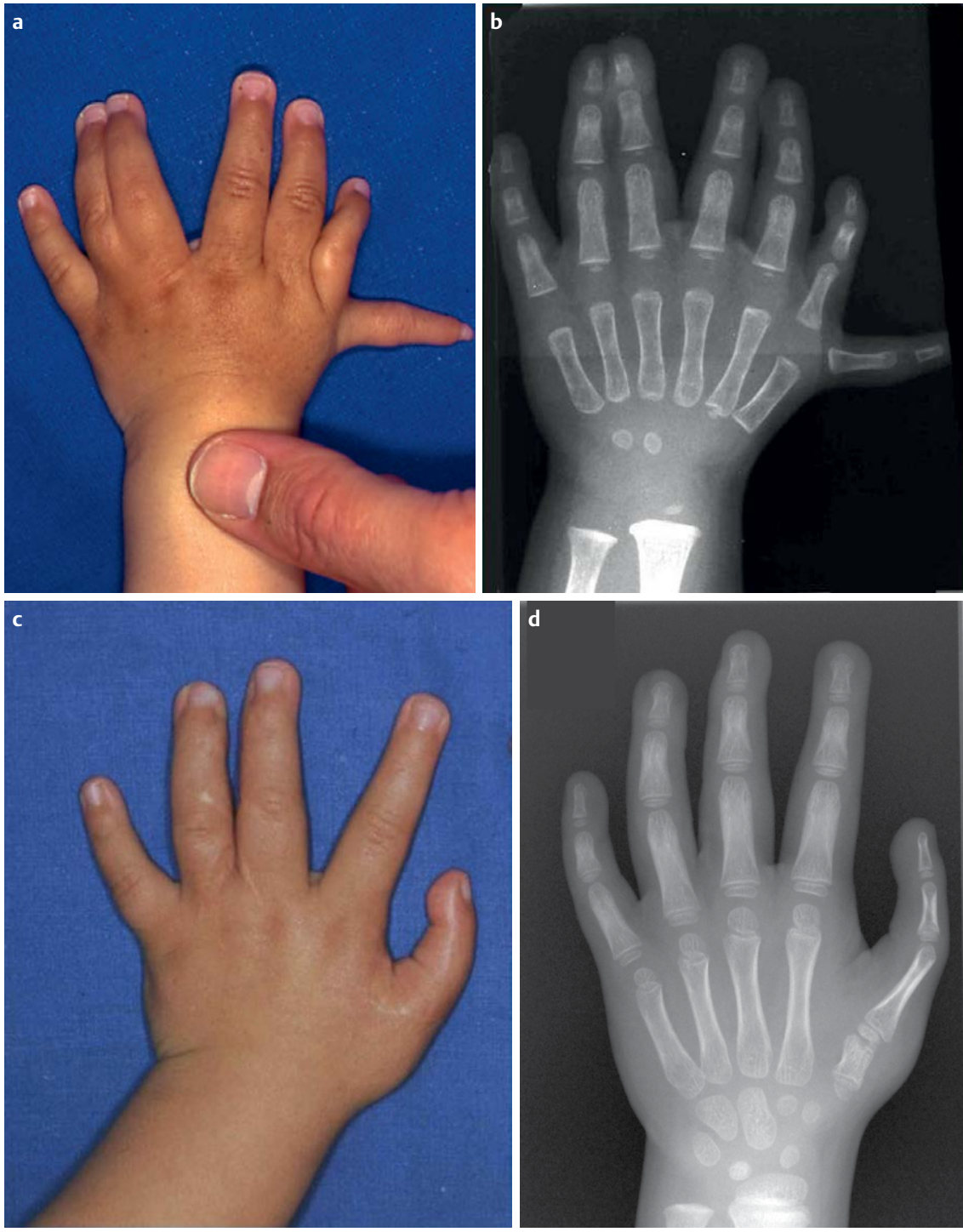

Abb. 10 Analyse der Veränderungen und Entwickeln der Behandlungsstrategie bei einem 2-jähriges Mädchen mit Polysyndaktylie. a präoperativer, klinischer Status: Welche Veränderungen liegen vor? Eine Syndaktylie ulnarer Finger und zu viele Finger auf der Radialseite; b präoperativer, radiologischer Status: In Daumenposition findet sich ein 3-gliedriger Finger und ein Doppeldaumen mit 3-gliedrigem ulnaren Anteil und sehr hypoplastischem radialen Anteil. Der zweite Mittelhandknochen von radial weist 2 Wachstumsfugen auf, eine distale und eine proximale. Da die sonstige Skelettanlange eher für einen Finger als für einen Daumen spricht, ist von einem Finger mit proximaler Pseudoepiphyse des Mittelhandknochens auszugehen; c klinischer Befund der Hand nach Resektion des hypoplastischen Doppeldaumens und Pollizisation des Fingers sowie Syndaktylietrennung von Mittel- und Ringfinger. $\mathbf{d}$ radiologischer Befund der Hand nach Resektion des hypoplastischen Doppeldaumens, Pollizisation des zweiten Fingers von radial und Syndaktylietrennung von Mittel- und Ringfinger. ger häutig syndaktyl miteinander verbunden. In Daumenposition findet sich ein 3-gliedriger Finger und ein Doppeldaumen. Der Doppeldaumen besteht aus einem 3-gliedrigen ulnaren Anteil und einem sehr hypoplastischen radialen Anteil.

Was benötigt das Kind, um besser greifen zu können? Es braucht einen funktionierender Daumen und voneinander getrennte Finger. Aus welchem Anteil und mit welcher Technik kann der beste Daumen gebildet werden? Der Doppeldaumen ist sehr hypoplastisch, der Finger deutlich besser entwickelt. Daher ist eine Resektion des hypoplastischen Doppeldaumens mit seinem Mittelhandknochen und eine Pollizisation des Fingers neben der Syndaktylietrennung von Mittel- und Ringfinger zu empfehlen (० Abb. 10c).

In der Fehlbildungschirurgie folgen nur wenige Operationen einem standardisierten Vorgehen. Standardisiert sind Syndaktylietrennungen, Pollizisationen, die Klumphandbehandlung und einfache Doppeldaumenkorrekturen. Immer muss intraoperativ mit überraschenden Pathologien gerechnet werden, die ein Abändern des operativen Vorgehens erfordern.

Wir sehen bei Kleinkindern eine wesentlich bessere Heilung als bei Erwachsenen. Allerdings weisen sie eine ausgeprägtere postoperative Schwellneigung auf. Nach zu ausgedehnten Opera- tionen können verstärkt durch die Schwellung auch bei Kleinkindern Wundheilungsstörungen und Weichteilnekrosen auftreten.

\section{Zusammenfassung \\ $\nabla$}

Beim ersten Kontakt mit Kindern mit Fehlbildungen schaut man, ob ein Syndrom vorliegt und ob eine mögliche Erblichkeit gegeben ist. Dann analysiert man, was an den Extremitäten verändert ist und überlegt, welche Änderungen notwendig sind, um die Funktion zu verbessern. Welche Techniken stehen hierfür zur Verfügung? Ziel der Behandlung ist, die bestmögliche Funktion bei akzeptablem Aussehen herzustellen. Von der Natur 


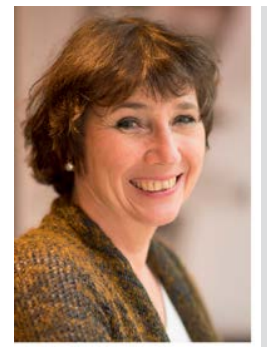

Wiebke Hülsemann, geb. 8.2.1957 in Tornesch, Schleswig-Holstein. Medizinstudium von 1975 bis 1982 in Kiel und Hamburg; allgemeinchirurgische Ausbildung von 1983 bis 1986 im Krankenhaus Föhr-Amrum (Dr. G. Valbert), chirurgische Weiterbildung von 1986 bis 1988 im Gemeinschaftskrankenhauses Herdecke (Dr. J. Möbius) und von 1991 bis 1996 in der Abteilung für Chirurgie und Gefäßchirurgie im Krankenhaus Itzehoe (Prof. Dr. N. Hantschmann). Unfallchirurgische Ausbildung im gleichen Haus von 1996 bis 1998 (Dr. K. Ehrke und Prof. Dr. Jürgens). Von 1988 bis 1991 handchirurgische Ausbildung im Krankenhaus Elim in Hamburg (Dr. R. Neumann). Promotion 1989, 1997 Fachärztin für Chirurgie, 1998 Teilgebietsbezeichnung Unfallchirurgie, 1997 Zusatzbezeichnung Handchirurgie. Von 1999 bis 2014 leitende Oberärztin in der Abteilung für Handchirurgie und angeborene Fehlbildungen im Kinderkrankenhaus Wilhelmstift (Dr. Rolf Habenicht), seit 1.9.2015 Chefärztin der Abteilung. nicht angelegte Finger kann ich durch die Transplantation von Zehen aufbauen. Zu kurze Handanteile können durch Kallusdistraktion verlängert und Gewebe durch Weichteildistraktion vermehrt werden.

Interessenkonflikt: Nein

\section{Literatur}

1 Flatt AE. The care of congenital hand anomalies. Classification and incidence. St. Louis, MO: Quality Medical Publishing; 1994: 47-63

2 Mundlos S, Horn D. Limb Malformations, Thalidomide Embryopathy. Berlin Heidelberg: Springer; 2014

3 Andersson GB, Gillberg C, Fernell E et al. Children with surgically corrected hand deformities and upper limb deficiencies: self-concept and psychological well-being. J Hand Surg Eur 2011; 36: 795-801

4 Buck-Gramcko D (Ed.) Congenital Malformations of the Hand and Forearm. Churchill Livingstone; London: 1998

\section{Dem interessierten Leser sei als weiterführende Literatur} empfohlen:

Gupta A, Kay SPJ, Scheker LR (Eds.). The Growing Hand. Mosby, London, 2000 Abzug JM, Kozin SH, Zlotolow DA (Eds.). The Pediatric Upper Extremity. Springer, New York, 2015 